\title{
A novel concept of human antiviral protection: It's all about RNA (Review)
}

\author{
TAMARA ARIPOVA $^{1 *}$ and JAVDAT MURATKHODJAEV ${ }^{1,2^{*}}$ \\ ${ }^{1}$ Institute of Immunology and Human Genomics, Academy of Sciences of Uzbekistan, Tashkent 100060; \\ ${ }^{2}$ GENEX LLC Pharmaceutical Company, Tashkent 100052, Uzbekistan
}

Received November 24, 2021; Accepted January 10, 2022

DOI: $10.3892 /$ br.2022.1512

\begin{abstract}
The comparative analysis of the antiviral protective mechanisms, including protozoa and RNA interference in multicellular organisms, has revealed their similarity and provided a basic understanding of adaptive immunity. The present article summarizes the latest studies on RNA-guided gene regulation in human antiviral protection, and its importance. Additionally, the role of both neutralizing antibodies and the interferon system in viral invasion is considered. The interferon system is an additional mechanism for suppressing viral infections in humans, which shifts cells into an 'alarm' mode to attempt to prevent further contagion. The primary task of the human central immune system is to maintain integrity and to protect against foreign organisms. In this review, a novel concept is proposed: Antiviral protection in all organisms can be achieved through an intracellular RNA-guided mechanism. A simple and effective defence against viruses is incorporation of a part of a virus's DNA (spacer) into the hosts chromosomes. Following reinfection, RNA transcripts of this spacer are created to direct nuclease enzymes to destroy the viral genome. This is an example of real-time adaptive immunity potentially possessed by every cell with a full complement of chromosomes, and an indicator that antiviral immunity is not only mediated by the presence of neutralizing antibodies and memory B-and T-cells, but also by the presence of specific spacers in the DNA of individuals who have recovered from a viral infection.
\end{abstract}

Correspondence to: Dr Javdat Muratkhodjaev, Institute of Immunology and Human Genomics, Academy of Sciences of Uzbekistan, 74 Ya. Gulyamov Street, Tashkent 100060, Uzbekistan E-mail: javdat_m@yahoo.com

*Contributed equally

Key words: RNA-I, CRISPR-Cas, antiviral immunity, interferon, COVID-19, SARS-CoV-2 spacers

\section{Contents}

1. Introduction

2. RNA-guided antiviral protection

3. The role of the interferon system

4. Discussion

5. Conclusions

\section{Introduction}

The current COVID-19 pandemic poses a substantial challenge for the entire medical community globally, and has spurred numerous studies to improve our understanding of the immune system. COVID-19, which can be detected by PCR and antibody titres has resulted in a widespread increase in the number of infected individuals, as well $>5$ million deaths, globally, and repeated lockdown measures to prevent or reduce the severity of outbreaks. This pandemic has highlighted a need to revisit some of the dogmas of immunology. One such dogma states that the memory of past infections is formed by $\mathrm{T}$ and B memory cells only. Several research articles have shown the presence of memory in innate nonspecific immunity, and this may provide a challenge to the prevailing point of view $(1,2)$. Conversely, the substantial amount of literature concerning gene regulation by RNA makes it possible to formulate a mechanism of human antiviral defence from a novel angle: Every cell in the human body with a full complement of chromosomes potentially has its own antiviral protection mechanism based on RNA interference.

To prove this point, the following sections of this review article describe the activity of RNA-guided gene regulation, and the role of interferons and the central immune system in viral invasion.

\section{RNA-guided antiviral protection}

To understand the mechanism of RNA-guided protection, it is necessary to start with unicellular organisms, as development of reliable mechanisms for countering viruses, the evolutionary acquisition of viral infection immunity allowed for the generation of multicellular organisms. It should be noted that this transition took $\sim 2$ billion years of evolution (the first multicellular organisms appeared 1.7 billion years 
ago $(3,4)$, and the first cells already existed 3.7-4.2 billion years ago $(5,6)$, as a result of which, multicellular organisms now possess the intracellular protection systems initially developed by bacteria and archaea, CRISPR-Cas. This system is adaptive and accumulates memory about previous encounters with viruses. This memory is stored in special DNA regions, which appear after a challenge with a foreign genome of the virus or plasmid in CRISPR arrays-short palindromic repeats, regularly arranged in groups (7). The virus entering a bacterial cell is detected by the CRISPR-associated (Cas) proteins, a type of nuclease that acts like a pair of scissors, to cut-out viral nucleic acid sequences. CRISPR-Cas is a real-time adaptive immune system with a memory of previous encounters with foreign viruses, that is stored in the unique spacer sequences obtained from the viral and plasmid genomes, and inserted into the CRISPR arrays (7). Spacer transcripts, together with other regions of the surrounding repeats, are used as guide RNAs to recognize related sequences in foreign genomes, and thus provide specific cleavage sites for Cas nucleases (8). This provides a simple and effective protective mechanism against viruses, whereby a part of the viral DNA (spacer) is inserted into the cell genome, and upon repeat infection with the same virus, a copy of this spacer, in the form of a small RNA, directs the nuclease enzymes to destroy the foreign genome (Fig. 1).

In multicellular organisms, there is a similar mechanism for regulating the activity of various genes termed RNA interference. Here, it is hypothesized that RNA interference is an essential component of the adaptive immune system in multicellular organisms, including humans. This system was first discovered in 1998 in Caenorhabditis elegans by Fire et al (9), who was subsequently awarded the Nobel Prize in Physiology or Medicine (2006). The mechanism of interference has already been studied in detail-it is widely used in experimental biology for knocking down certain genes, and in medicine for treatment of certain types of cancer (10-12).

The interference itself consists of halting the translation of viral genes by cutting or modifying them $(13,14)$. For this, the cells have a special complex of nuclease enzymes, which are controlled by small RNAs-the same transcript spacers. Insertion of the spacer into the DNA of the cell itself is the final 'vaccination' stage of the target cell after viral invasion. When the virus enters the cell again, the small RNAs are synthesized and loaded into the nuclease complex to direct cutting of the foreign genome (Fig. 1). Thus, there is a complete analogy between these two systems of RNA-the guided antiviral immunity of cells by RNA. At present, it is unclear how certain regions of the viral material are incorporated into the cell's DNA. However, the very existence of such mechanisms has been described in studies on retrotransposons and pseudogenes $(15,16)$, where intracellular reverse transcriptase converts cytoplasmic RNA and transcribes retroelements into complementary DNA. Human telomerase, which is essentially a reverse transcriptase, actively uses proteins involved in RNA interference to synthesize telomeres with their subsequent integration into the DNA of chromosomes. It should be noted that retroelements make up a half of the human DNA $(17,18)$, and it is logical to assume that a significant part of the human genome has encoded some DNA fragments of previously encountered viral genomes-those very spacers (19). Moreover, this assumption has already been proven by the presence of SARS-CoV-2 spacers in DNA of infected people (20).
The role of RNA interference has been proven to occur in several infections caused by the human respiratory syncytial virus (21), human immunodeficiency virus type 1 (22), hepatitis B virus (23) hepatitis C virus $(24,25)$, influenza virus (26) and coronavirus SARS-CoV-1 (27). The presence of such spacers effectively prevents viral infection in mammals as well. It is known that the spacers in the DNA of target cells inhibit the reproduction of viruses $(28,29)$. Recent work on the suppression of SARS-CoV-2 viral reproduction using specific siRNAs (30) leaves no doubt regarding the validity of this hypothesis.

The data mentioned above directly indicate the ability of cells themselves to resist viral invasion. Every cell in the human body that contains a full complement of chromosomes may potentially preserve an ancient system for counteracting viruses using small RNAs. Moreover, this protection is adaptive and forms a type of full-fledged intracellular immune memory.

\section{The role of the interferon system}

The interferon system is another important mechanism for cellular protection, which is based on production of special proteins preventing further infection $(31,32)$. It is hypothesized that this additional system is required for highly organized organisms to respond quickly to a viral invasion. The increase in the number of densely grouped cells of the same type facilitates the spread of viruses-having multiplied in one sensitive cell, virions can easily infect the nearby cells. Accordingly, the innate RNA-guided protection may not be able to cope with the high viral load. To prevent this possibility, an early warning system exists and uses interferons as 'alarms'.

All nucleated cells have receptors for interferon I (33). Following activation of this receptor upon ligand binding, the expression of several genes is upregulated placing the cell in a state of 'alarm', halting almost all protein synthesis, and endo- and exocytosis are inhibited, which prevents both entry and exit of viral particles $(34,35)$. Interferon itself is produced by cells infected with viruses (36). Each human cell possesses a substantial profile of receptors that recognize certain pathogenic motifs. In the case of viral infections, there are special cytoplasmic RLR receptors that recognize viral double-stranded RNA (37). Their activation triggers a cascade of intracellular mechanisms, ultimately resulting in the synthesis of interferons and pro-inflammatory cytokines (Fig. 2).

Interferons themselves, in turn, regulate the functions of $>2,000$ interferon stimulated genes (ISGs); there is a database of these ISGs, which highlights their effect on metabolism (interferome.org/interferome/home.jspx). In the context of this review, only a few points will be highlighted. First, the epithelial cells, which are the first to meet various pathogens, possess receptors for interferon III. This emphasizes the need for prompt and coordinated activity during viral invasions of these barrier forming cells. Second, prolonged contact with interferons induces the cells to initiate apoptosis. Finally, several enzymes are produced by interferon blocking nucleases, which are required for the full function of the RNA-guided system (38). Thus, an additional safety interferon system is able to interfere with the RNA-controlled protection and, moreover, induce cell 


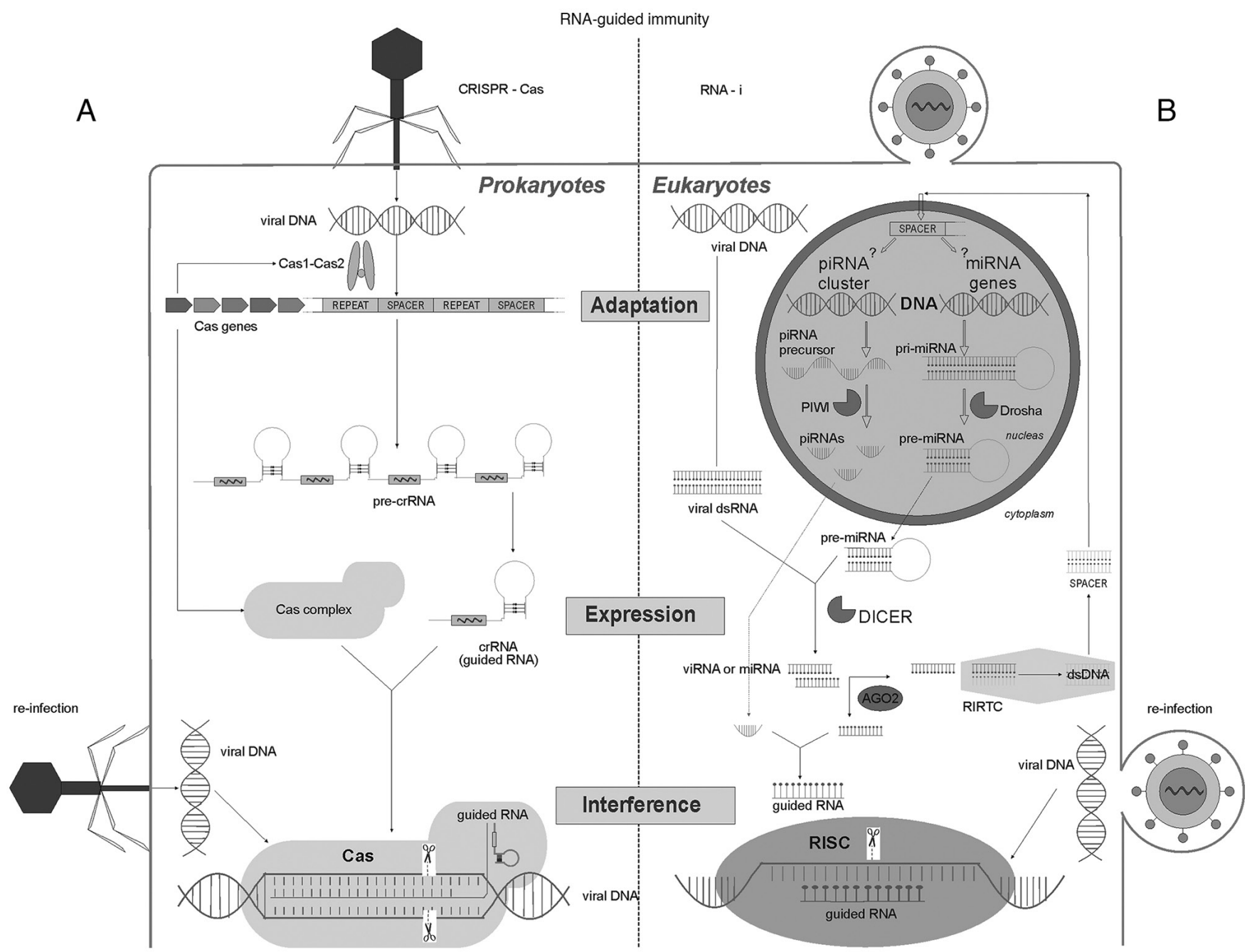

Figure 1. Key stages of RNA-guided immunity-adaptation, expression and interference of the antiviral defence systems of protozoa and humans. (A) The left side shows the immune system of bacteria and archaea, including the CRISPR-Cas system. During adaptation, phage DNA is cleaved by Cas1/Cas2 enzymes into spacers and integrated into a special CRISPR locus. A specific adaptive memory is formed based on a previous infection. Upon re-infection with the same virus, RNA transcripts from these spacers are directed to complexes formed by other Cas nucleases (the CRISPR-Cas9 complex is shown here), where they serve as a template for enzymes that are used to cut similar nucleotide sequences in the viral genome. (B) Stages of RNA interference in human cells. When cells are infected with RNA or DNA viruses (DNA polymerases of viruses generate RNA sequences), long double-stranded RNAs are formed in the cytoplasm, which are cut by DICER nucleases into short double-stranded viRNAs. Thereafter, the AGO2 enzyme unwinds the viRNA strands and loads them either into an RISC or into an RIRTC. In the RISC, the viral RNAs are cleaved, and in the RIRTC a spacer is synthesized-a double-stranded DNA molecule from the RNA template. It is hypothesized that this involves a reverse transcription complex similar to the telomerase TERT, in which the AGO2 protein directs the RNA sequence to form new DNA telomeres (62). The spacer integrates into the DNA of the cell, forming a specific memory of a past infection. It remains unclear where exactly in human chromosomes spacer sequences are formed in piRNA clusters or at miRNA loci. When viruses enter this cell again, the spacer is transcribed into a pri-miRNA, which, under the action of DROSHA is shortened to a pre-miRNA and shuttled to the cytoplasm after DICER-mediated cutting, where it is loaded by AGO2 into a RISC, and serves as a template for cleaving viral RNAs. piRNA, piwi-interacting RNA; miRNA, microRNA; dsRNA, double stranded RNA; dsDNA, double stranded DNA; viRNA, viral short PHK; PIWI, P-element Induced WImpy testis; DROSHA, Drosha Ribonuclease III; DICER, endoribonuclease Dicer; AGO-2, Argonaute RISC Catalytic Component 2; RISC, RNA induced silencing complex; RIRTC, RNA induced reverse transcription complex; crRNA, CRISPR RNA; Cas, CRISPR associated.

death. This particular kind of antagonism in the interferon and RNA-guided systems in antiviral protection has been studied in several studies, and is extensively reviewed here (39). The first cell lines that exhibit viral entry are well-differentiated surface epithelial or endothelial cells. When the virus enters these cells, DICER nucleases begin immediately cutting the viral genome, the TLR and RLR receptors, and in turn, trigger a cascade of interferon synthesis $(37,38)$. This is the first non-specific phase of countering the viral invasion. The following steps are dependent on the viral load; if it is small, then the infected cells cope on their own and 'warn' the neighbouring intact cells about the infection, assisting the latter in reducing their susceptibility to viral infection. The infection is interrupted without a pronounced clinical effect. As the load increases, the infected cells undergo apoptosis, and the neighbouring cells, under the influence of increasing doses of interferon, halt protein synthesis, exo- and endocytosis, and the activity of almost all enzymes; these cells metaphorically freeze $(33,34,40)$. Under the influence of interferons and other cytokines, the central immune system is primed; the first antibodies and symptoms of inflammation appear. The infected cells carrying viral antigens on their surface to attract T-killers, these cells are opsonized with antibodies, and the complement system is activated $(41,42)$. The classical signs of 


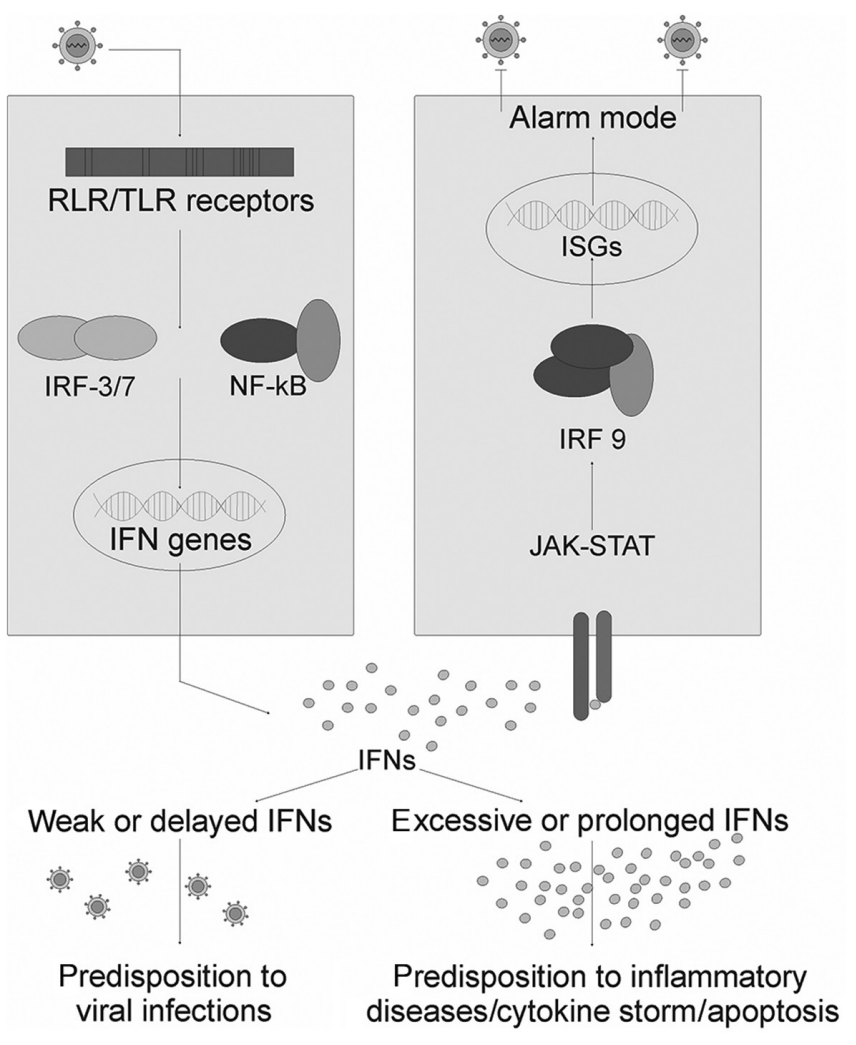

Figure 2. The Interferon alarm system: Adapted from Onomoto et al (40). Activation of the cytoplasmic receptors RIG-1 and MDA5 by viral dsRNAs, as well as endosomal receptors TLR 7/8 by ssRNAs, lead to phosphorylation of IRF3 and 7, and the pro-inflammatory factor NF-kB. These transcription factors induce the expression of interferon genes and inflammatory cytokines upon nuclear translocation. Newly synthesized interferons bind to their receptors on neighbouring cells and induce the expression of ISGs through the Jak/STAT pathway. Under the influence of the restriction factors encoded by these genes, the cell goes into an 'alarm' mode, and in this state, they halt protein synthesis, including that of viral proteins, and marks all newly synthesized proteins for degradation. Vesicular transport is slowed, which leads to inhibition of virion assembly and release. Excessive production of IFN induces cell apoptosis and is associated with the development of a cytokine storm. During a cytokine storm, there is the risk of the formation of autoimmune attacks. The lack of IFN production does not prepare cells for viral invasion and, as a result, exacerbates an infectious disease. RIG-1, retinoic acid inducible gene-1; MDA5, melanoma differentiation associated protein-5; TLR, toll-like receptor; IFN, interferon; IRF, IFN regulatory factor; NF- $\mathrm{\kappa B}$, nuclear factor- $\mathrm{\kappa B}$; JAK, Janus kinase; STAT, Signal Transducer and Activator of Transcription proteins; ISG, IFN stimulated gene; ssRNA, single stranded RNA.

an infection attributed to each specific virus manifest. Note, while the viral proteins are presented at the plasma membrane of infected cells, the immune system is in an activated state. Moreover, the cells presenting these viral antigens are eventually destroyed.

Concurrently, in the basal layers of these tissues, where actively proliferating and unipotent cells are located, when the viruses and/or spacers themselves enter from the infected cells, an adaptive intracellular antiviral protection begins to form. As mentioned above, poorly differentiated cells, under the influence of interferons, do not halt RNA and protein synthesis (43). Accordingly, there remains a place for full-fledged RNA interference, which is not disturbed by blocking interferon signals. Therefore, immune memory is formed in unipotent progenitor cells when a virus or spacer
RNA penetrates them through extracellular vesicles or nucleoprotein complexes with AGO $2(44,45)$. After maturation, these cells will possess specific antiviral memory, providing them a powerful tool to effectively eliminate any further infections with the same virus $(28,29)$. The newly formed layer of cells readily copes with the residual viral load and no longer carries the antigens of the virus; the central immune system returns to a normal mode once stimulation is gradually decreased. This change in endothelial and epithelial cells usually takes several days, which is the time required for formation of specific local antiviral memory.

Thus, it is hypothesized that every cell of the human body with a full complement of chromosomes potentially possesses this form of antiviral protection; and it is the RNA interference response that initially determines the course of a viral infection.

\section{Discussion}

The CRISPR-Cas system has proven to be incredibly effective in combating mobile genetic elements, and thus has retained its role in multicellular organisms, having slightly changed, taking into account the presence of a nuclear membrane and terminal chromosomes. The primary goal of the central immune system possessed by higher order animals, based on $\mathrm{T}$ and $\mathrm{B}$ cells, is to maintain the integrity of the organism and counter foreign organisms. To accomplish this, the immune system possesses phagocytic, regulatory, antigen-presenting and killer cells, as well as a complement system, and of course, various antibodies. The complement system is a group of proteins, which, after being activated, promote membranolytic cascades that destroy target cells. The classical pathway is activated when the Clq complement protein binds to the Fc-fragment of the antibody (this is the invariable part of all antibodies in the body) attached to the antigen (42). It should be emphasized that antibodies do not destroy a foreign object by themselves, even in high concentrations, they perform a diagnostic and guiding role only. In a multi-trillion cell human body, the antibodies help immune cells to identify foreign agents by binding to specific foreign antigens (opsonization) (46). Namely, the Fc-fragment of antibodies is a 'black mark' for immune cells and complement, which perceive it as a signal to destroy this object (41). In the case of bacteria, protozoa, fungi and helminths, this is a working strategy by which they are destroyed through the use of antibodies. This also works to counter mutant cells, including tumour cells, where antibodies bind to cancer antigens, and such cells are also discarded. But what happens in the case of a viral infection? A priori, viruses multiply inside cells only, and when viral antigens present on the cell membrane are detected by antibodies, these infected cells are also destroyed. For the entire organism, this is incredibly beneficial; when something foreign is identified, even inside their own cells, the foreign body and/or affected cell is destroyed, sacrificing a part for the greater good of the whole. Note that these infected cells have no other fate-they are all destroyed.

Until the viruses have penetrated the cells, they are just a set of nucleic acids and proteins; they do not multiply, and do not possess any pathological effects on the body. When viruses bind with antibodies, they of course lose their 
ability to target cells, making it difficult for them to bind to cellular receptors. This is the rationale behind the vaccination strategy. However, let us consider a further course of events; the resulting virus-antibody complex must be destroyed by immune cells carrying the receptor for the Fc fragment. In several cases, this happens, but if phagocytosis is not effective, and the virus remains viable inside these cells, the infection intensifies further, since the virus has infected cells that do not carry receptors to it, and this phenomenon is called antibody-dependent enhancement (ADE) (47-49). In the case of ADE, the virus infects susceptible cells through the corresponding receptor and penetrates monocytes/macrophages, granulocytes, platelets, mast cells and several other host cells by interacting with receptors for the Fc fragment or complement (50). There are several examples of ADE caused by $\alpha$ and $\beta$ coronaviruses $(51,52)$. The current clinical data on the course of COVID-19 indicate involvement of antibodies in the enhancement of clinical manifestations of the disease. The most severe patients appear to possess the highest antibody titres (53). A specific symptom of COVID-19, coagulopathy, clearly indicates complement hyperactivity (54); and this process of cell destruction does not stop as long as they carry viral antigens on their surface. Only as a result of RNA-guided nucleases does the cell achieve clearance of the viral genome, and, accordingly, the viral proteins. Further activation of the central immune system stops, antibody titres fall, and the affected individual recovers.

While discussing human antiviral immunity, one cannot fail to mention the mechanisms of protection observed in early childhood. As recent data have shown, along with antibodies, breast milk contains $\sim 1,400$ different types of microRNAs (55). Given the ability of each of these molecules to alter the activity of an average of 15-20 genes, there is a tremendous opportunity to suppress or enhance the activity of genes in infants. It has been shown that these microRNAs, after absorption, are present in the bloodstream and all tissues of the body, including the brain $(56,57)$. Thus, with regards to antiviral immunity, it is necessary to emphasize the presence of such a transfer of protection through milk to a child.

The role of vitamin A in prevention and treatment of viral diseases should also be mentioned. There is a positive association between vitamin A administration and the management of measles. During a measles infection, it has been shown that vitamin A deficiency clearly correlates with the severity of the course, and timely treatment of measles with two doses of retinol (200,000 IU) dramatically reduces both morbidity and mortality rates (58-60). We hypothesize that there may be a possibility of wider use of this simple and cheap drug for other viral diseases, including COVID-19. This vitamin is undoubtedly important for the synthesis of RLR receptors, but we were interested in the fact that the DICER nuclease and the RLR receptor have a similar structure, they both possess a DECH box domain that recognizes viral RNA (61). DICER nuclease is a key player in RNA-driven gene regulation, and further research is required on the possible relationship between Vitamin A and RNA interference.

From the above concept of cellular adaptive antiviral immunity, several assumptions can follow regarding interpretation of clinical indicators during the course of a viral infection. Taking COVID-19 as an example, they are as follows: i) The presence or absence of specific antibodies to SARS-CoV-2 is not a predictor of the disease. The presence of antibodies in the blood reflects only the fact that a person has been in contact with the virus. Lack of antibodies does not mean any contact, and people with high titres of specific antibodies are not protected from re-infection with SARS-Co2. ii) PCR tests for those who have had COVID may return false positives if the swab sample is taken from the point of the initial spread of the virus (usually from the nasopharynx). We suggest that a negative PCR result for COVID in the blood plasma and urine may be a more reliable indicator of a lack on infection, even when a swab sample from the nasopharynx returns a positive result.

\section{Conclusions}

Here, a novel concept is proposed-the antiviral protection of all organisms based on intracellular RNA-guided mechanisms. A simple and effective defence against viruses is contained as part of the virus's DNA (spacer) in the chromosomes. Following a reinfection, the RNA transcribes the incorporated spacer and directs nuclease enzymes to cut the viral genome. This is a real-time adaptive immune response potentially possessed by every cell that contains a nucleus. Thus, antiviral immunity may not only be mediated by neutralizing antibodies and memory B- and T-cells, but also through the incorporation of specific spacers into the DNA of the cells genome.

\section{Acknowledgments}

We would like to thank Dr S. Muratkhodjaeva (Institute of Immunology and Human Genomics, Academy of Sciences of Uzbekistan, Tashkent, Uzbekistan) for her skilful drawings.

\section{Funding}

No funding was received.

\section{Availability of data and materials}

Not applicable.

\section{Authors' contributions}

TA and JM both wrote and revised the manuscript. Both authors have read and approved the final manusript. Data authentication is not applicable.

\section{Ethics approval and consent to participate}

Not applicable.

\section{Patient consent for publication}

Not applicable.

\section{Competing interests}

The authors declare that they have no competing interests. 


\section{References}

1. Reimer-Michalski EM and Conrath U: Innate immune memory in plants. Semin Immunol 28: 319-327, 2016.

2. Netea MG, Quintin J and van der Meer JW: Trained immunity: A memory for innate host defense. Cell Host Microbe 9: 355-361, 2011

3. Rosing MT: 13C-Depleted carbon microparticles in $>3700-\mathrm{Ma}$ sea-floor sedimentary rocks from west greenland. Science 283 : 674-676, 1999.

4. Dodd MS, Papineau D, Grenne T, Slack JF, Rittner M, Pirajno F, O'Neil J and Little CT: Evidence for early life in Earth's oldest hydrothermal vent precipitates. Nature 543: 60-64, 2017.

5. Knoll AH, Javaux EJ, Hewitt D and Cohen P: Eukaryotic organisms in Proterozoic oceans. Philos Trans R Soc Lond B Biol Sci 361: 1023-1038, 2006.

6. Fedonkin MA: The origin of the Metazoa in the light of the Proterozoic fossil record. Paleontol Res 7: 9-41, 2003.

7. Barrangou R: The roles of CRISPR-Cas systems in adaptive immunity and beyond. Curr Opin Immunol 32: 36-41, 2015.

8. Koonin EV and Makarova KS: Mobile genetic elements and evolution of CRISPR-Cas systems: All the way there and back. Genome Biol Evol 9: 2812-2825, 2017

9. Fire A, Xu S, Montgomery MK, Kostas SA, Driver SE and Mello CC: Potent and specific genetic interference by double-stranded RNA in Caenorhabditis elegans. Nature 391: 806-811, 1998

10. Elbashir SM, Harborth J, Lendeckel W, Yalcin A, Weber K and Tuschl T: Duplexes of 21-nucleotide RNAs mediate RNA interference in cultured mammalian cells. Nature 411: 494-498, 2001

11. Han H: RNA interference to knock down gene expression. Methods Mol Biol 1706: 293-302, 2018.

12. Abdelrahim M, Safe S, Baker C and Abudayyeh A: RNAi and cancer: Implications and applications. J RNAi Gene Silencing 2: 136-145, 2006.

13. Ghildiyal $M$ and Zamore PD: Small silencing RNAs: An expanding universe. Nat Rev Genet 10: 94-108, 2009.

14. Maillard PV, Ciaudo C, Marchais A, Li Y, Jay F, Ding SW and Voinnet O: Antiviral RNA interference in mammalian cells Science 342: 235-238, 2013.

15. Habibi L and Salmani H: Pivotal impacts of retrotransposon based invasive RNAs on evolution. Front Microbiol 8: 1957, 2017.

16. Wei W, Morrish TA, Alisch RS and Moran JV: A transient assay reveals that cultured human cells can accommodate multiple LINE-1 retrotransposition events. Anal Biochem 284: 435-438, 2004.

17. Lander ES, Linton LM, Birren B, Nusbaum C, Zody MC, Baldwin J, Devon K, Dewar K, Doyle M, FitzHugh W, et al: Initial sequencing and analysis of the human genome. Nature 409: 860-921, 2001

18. Wicker T, Sabot F, Hua-Van A, Bennetzen JL, Capy P, Chalhoub B, Flavell A, Leroy P, Morgante M, Panaud O, et al: A unified classification system for eukaryotic transposable elements. Nat Rev Genet 8: 973-982, 2007.

19. Javdat M and Tamara A: RNA interference: Antiviral defense mechanism and immune memory. Adv Appl Physiol 5: 24-29, 2020.

20. Zhang L, Richards A, Barrasa MI, Hughes SH, Young RA and Jaenisch R: Reverse-transcribed SARS-CoV-2 RNA can integrate into the genome of cultured human cells and can be expressed in patient-derived tissues. Proc Natl Acad Sci USA 118: e2105968118, 2021.

21. Bitko V and Barik S: Phenotypic silencing of cytoplasmic genes using sequence-specific double-stranded short interfering RNA and its application in the reverse genetics of wild type negative-strand RNA viruses. BMC Microbiol 1: 34, 2001.

22. Coburn GA and Cullen BR: Potent and specific inhibition of human immunodeficiency virus type 1 replication by RNA interference. J Virol 76: 9225-9231, 2002.

23. McCaffrey AP, Nakai H, Pandey K, Huang Z, Salazar FH, Xu H, Wieland SF, Marion PL and Kay MA: Inhibition of hepatitis B virus in mice by RNA interference. Nat Biotechnol 21: 639-644, 2003.

24. Yokota T, Sakamoto N, Enomoto N, Tanabe Y, Miyagishi M Maekawa S, Yi L, Kurosaki M, Taira K, Watanabe M and Mizusawa $\mathrm{H}$ : Inhibition of intracellular hepatitis $\mathrm{C}$ virus replication by synthetic and vector-derived small interfering RNAs. EMBO Rep 4: 602-608, 2003.

25. Krönke J, Kittler R, Buchholz F, Windisch MP, Pietschmann T, Bartenschlager R and Frese M: Alternative approaches for efficient inhibition of hepatitis $\mathrm{C}$ virus RNA replication by small interfering RNAs. J Virol 78: 3436-3446, 2004.
26. Ge Q, McManus MT, Nguyen T, Shen CH, Sharp PA, Eisen HN and Chen J: RNA interference of influenza virus production by directly targeting mRNA for degradation and indirectly inhibiting all viral RNA transcription. Proc Natl Acad Sci USA 100: 2718-2723, 2003.

27. He ML, Zheng B, Peng Y, Peiris JS, Poon LL, Yuen KY, Lin MC, Kung HF and Guan Y: Inhibition of SARS-associated coronavirus infection and replication by RNA interference. JAMA 290: 2665-2666, 2003.

28. Fujino K, Horie M, Honda T, Merriman DK and Tomonaga K: Inhibition of Borna disease virus replication by an endogenous bornavirus-like element in the ground squirrel genome. Proc Natl Acad Sci USA 111: 13175-13180, 2014.

29. Honda T and Tomonaga K: Endogenous non-retroviral RNA virus elements evidence a novel type of antiviral immunity. Mob Genet Elements 22: e1165785, 2016.

30. Idris A, Davis A, Supramaniam A, Acharya D, Kelly G, Tayyar Y, West N, Zhang P, McMillan CLD, Soemardy C, et al: A SARS-CoV-2 targeted siRNA-nanoparticle therapy for COVID-19. Mol Ther 29: 2219-2226, 2021.

31. Stetson DB and Medzhitov R: Type I interferons in host defense. Immunity 25: 373-381, 2006

32. Levy DE: Whence interferon? Variety in the production of interferon in response to viral infection. J Exp Med 195: 15-18, 2002.

33. de Weerd NA and Nguyen T: The interferons and their receptors-distribution and regulation. Immunol Cell Biol 90: 483-491, 2012

34. Houglum JE: Interferon: Mechanisms of action and clinical value. Clin Pharm 2: 20-28, 1983.

35. McNab F, Mayer-Barber K, Sher A, Wack A and O'Garra A: Type I interferons in infectious disease. Nat Rev Immunol 15: 87-103, 2015

36. Katze MG, He Y and Gale M Jr: Viruses and interferon: A fight for supremacy. Nat Rev Immunol 2: 675-687, 2002

37. $\mathrm{Wu} \mathrm{J}$ and Chen $\mathrm{ZJ}$ : Innate immune sensing and signaling of cytosolic nucleic acids. Annu Rev Immunol 32: 461-488, 2014.

38. van der Veen AG, Maillard PV, Schmidt JM, Lee SA, Deddouche-Grass S, Borg A, Kjær S, Snijders AP and Reis e Sousa C: The RIG-I-like receptor LGP2 inhibits Dicer-dependent processing of long double-stranded RNA and blocks RNA interference in mammalian cells. EMBO J 37: e97479, 2018

39. Maillard PV, van der Veen AG, Poirier EZ and Reis e Sousa C Slicing and dicing viruses: Antiviral RNA interference in mammals. EMBO J 38: e100941, 2019.

40. Onomoto K, Onoguchi K and Yoneyama M: Regulation of RIG-I-like receptor-mediated signaling: Interaction between host and viral factors. Cell Mol Immunol 18: 539-555, 2021.

41. Flesch BK and Neppert J: Functions of the Fc receptors for immunoglobulin G. J Clin Lab Anal 14: 141-156, 2000.

42. Duncan AR and Winter G: The binding site for C1q on IgG. Nature 332: 738-740, 1988.

43. Eggenberger J, Blanco-Melo D, Panis M, Brennand KJ and tenOever BR: Type I interferon response impairs differentiation potential of pluripotent stem cells. Proc Natl Acad Sci 116 1384-1393, 2019

44. Holtzman J and Lee H: Emerging role of extracellular vesicles in the respiratory system. Exp Mol Med 52: 887-895, 2020.

45. Geekiyanage H, Rayatpisheh S, Wohlschlegel JA, Brown R Jr and Ambros V: Extracellular microRNAs in human circulation are associated with miRISC complexes that are accessible to anti-AGO2 antibody and can bind target mimic oligonucleotides. Proc Natl Acad Sci USA 117: 24213-24223, 2020.

46. Mevorach D: Opsonization of apoptotic cells. Implications for uptake and autoimmunity. Ann N Y Acad Sci 926: 226-235, 2000

47. Hawkes RA: Enhancement of the infectivity of arboviruses by specific antisera produced in domestic fowls. Aust J Exp Biol Med Sci 42: 465-482, 1964

48. Smatti MK, Al Thani AA and Yassine HM: Viral-induced enhanced disease illness. Front Microbiol 9: 2991, 2018.

49. Tirado SM and Yoon KJ: Antibody-dependent enhancement of virus infection and disease. Viral Immunol 16: 69-86, 2003.

50. Khandia R, Munjal A, Dhama K, Karthik K, Tiwari R, Malik YS, Singh RK and Chaicumpa W: Modulation of dengue/zika virus pathogenicity by antibody-dependent enhancement and strategies to protect against enhancement in zika virus infection. Front Immunol 9: 597, 2018

51. Wan Y, Shang J, Sun S, Tai W, Chen J, Geng Q, He L, Chen Y, Wu J, Shi Z, et al: Molecular mechanism for antibody-dependent enhancement of coronavirus entry. J Virol 94: e02015-19, 2020.

52. Yip MS, Cheung CY, Li PH, Bruzzone R, Peiris JSM and Jaume M: Investigation of antibody-dependent enhancement (ADE) of SARS coronavirus infection and its role in pathogenesis of SARS. BMC Proc 5 (Suppl 1): P80, 2011. 
53. Chen X, Pan Z, Yue S, Yu F, Zhang J, Yang Y, Li R, Liu B, Yang X, Gao L, et al: Disease severity dictates SARS-CoV-2-specific neutralizing antibody responses in COVID-19. Signal Transduct Target Ther 5: 180, 2020.

54. Lo MW, Kemper C and Woodruff TM: COVID-19: Complement, coagulation, and collateral damage. J Immunol 205: 1488-1495, 2020.

55. Benmoussa A and Provost P: Milk MicroRNAs in health and disease. Compr Rev Food Sci Food Saf 18: 703-722, 2019.

56. Manca S, Upadhyaya B, Mutai E, Desaulniers AT, Cederberg RA, White BR and Zempleni J: Milk exosomes are bioavailable and distinct microRNA cargos have unique tissue distribution patterns. Sci Rep 8: 11321, 2018.

57. Zempleni J: Milk exosomes: Beyond dietary microRNAs. Genes Nutr 12: 12, 2017.

58. Soye KJ, Trottier C, Richardson CD, Ward BJ and Miller WH Jr: RIG-I is required for the inhibition of measles virus by retinoids. PLoS One 6: e22323, 2011.
59. D'Souza RM and D'Souza R: Vitamin A for the treatment of children with measles-a systematic review. J Trop Pediatr 48: 323-327, 2002.

60. Huiming Y, Chaomin W and Meng M: Vitamin A for treating measles in children. Cochrane Database Syst Rev 2005: CD001479, 2005

61. Paro S, Imler JL and Meignin C: Sensing viral RNAs by Dicer/RIG-I like ATPases across species. Curr Opin Immunol 32: 106-113, 2015.

62. Laudadio I, Orso F, Azzalin G, Calabrò C, Berardinelli F, Coluzzi E, Gioiosa S, Taverna D, Sgura A, Carissimi C and Fulci V: AGO2 promotes telomerase activity and interaction between the telomerase components TERT and TERC. EMBO Rep 20: e45969, 2019. 